\title{
Incidental findings detected with panoramic radiography: prevalence calculated on a sample of 2017 cases treated at a major Italian trauma and cancer centre
}

\author{
Sogol Ghassemzadeh ${ }^{1} \cdot$ Luca Sbricoli $^{1} \cdot$ Anna Chiara Frigo $^{2} \cdot$ Christian Bacci $^{1}$ (i)
}

Received: 22 May 2020 / Accepted: 21 October 2020 / Published online: 19 November 2020

(c) The Author(s) 2020

\begin{abstract}
Objectives This study aimed to assess the prevalence of incidental findings, not strictly related to dentistry, viewed with panoramic radiography.

Methods Panoramic radiographs performed between December 2013 and June 2016 were retrospectively collected. These images were analyzed, searching for incidental findings. All the information collected was statistically analysed

Results A total of 2307 Panoramic Radiograph were analyzed and 2017 of them were included in the study. 529 incidental findings were seen: 255 (48.2\%) were ESP (Elongation of Styloid Process), 167 were CAC (Carotid Artery Calcification) (31.57\%), 36 were maxillary sinus pathologies (6.8\%) and 71 were other incidental findings (13.42\%). The total prevalence of IF was $26,23 \%$., CAC was $8.28 \%$ in the total population, and it was higher in women (9.82\%) than men (6.54\%). $48.5 \%$ of CAC were bilateral. When unilateral, the right side showed a higher right side prevalence. The prevalence of ESP was $12.64 \%$ in total population (men: $13.82 \%$; women: $11.60 \%$ ). $84.71 \%$ of ESP were bilateral and, when present unilaterally, no side difference was seen. $13.33 \%$ of the ESP appeared segmented. The prevalence of maxillary sinus pathologies was $1.78 \%$ (men: $2.32 \%$; women: $1.31 \%$ ). Only $8.33 \%$ of these pathologies were bilateral, and, when unilateral, they were mostly present on the right side. Between the 71 other IF (prevalence: $3.52 \%$ ), sialoliths and tonsilloliths were assessed most frequently.

Conclusion Due to the high prevalence of incidental findings detected with panoramic radiography, dental practitioners should be aware of the various pathologic conditions seen on the panoramic radiographs.
\end{abstract}

Keywords Panoramic radiography $\cdot$ Incidental findings $\cdot$ Atherosclerosis $\cdot$ Carotid artery calcification $\cdot$ Elongation of styloid process $\cdot$ Maxillary sinus

\section{Aim}

The present study aimed to estimate the prevalence of incidental findings, detected using Panoramic Radiograph (PR) examinations, with the exception of caries, periodontal and periapical diseases.

Christian Bacci

christian.bacci@unipd.it

1 Unit of Oral Medicine, Pathology and Surgery, Section of Clinical Dentistry, Department of Neurosciences, University of Padova, Via Giustiniani, 1, 35128 Padova, Italy

2 Department of Public Health, University of Padova, Padova, Italy
Evaluation of PRs, in assessing carotid artery calcification, elongated stylohyoid process, maxillary sinus pathologies, and any other incidental findings, was made.

\section{Materials and methods}

A consecutive case series of panoramic radiographs performed in the University Hospital of Padova between December 2013 and June 2016 were retrospectively collected. The study was performed in full accordance with the World Medical Association Declaration of Helsinki since none of the patients underwent any radiography for this study. All the patients needed panoramic radiographs for dental treatment procedures, trauma, and necessity to start bisphosphonates therapy, to undergo a transplant or cardiac intervention. Therefore, the bias has been reduced because 
none of these PRs were taken to highlight the anomalies described in the aim of this study.

The panoramic radiographs had been made using the same digital panoramic system. The PRs were examined by 3 expert dentists working in the University of Padova. Finally, a radiologist, who was unaware of the intent of the study, approved all the findings. During the examination of the radiographs, no time limit was imposed.

The PRs were observed in full-screen mode. Overexposed images or calcifications that had low radiopacity were clarified or zoomed in using software tools. If a patient had multiple PRs, only one of them was included in the study, choosing the one with the best image quality. Radiographs that were distorted because of the subjects' movements during the exposure and those that did not include $\mathrm{C} 3$ and $\mathrm{C} 4$ vertebrae were excluded. Images with an ambiguous diagnosis due to superimposed structures or lack of clarity, were discarded as well. PRs that did not display the stylohyoid ligament complex in good image quality or in which the origin of the styloid process from the lower part of the temporal bone was hidden by the shadows of the base of the skull, were not included in the study. The presence of radiolucent lesions and radiopacities compatible with mineralization were studied, as well as their unilateral or bilateral characteristics. The patient's age and sex were recorded for each radiograph reviewed.

\section{(a) Carotid artery calcifications}

Since their presence was first reported in 1981 [1], radiopacities in the lateral parts of the neck, have received more attention. In fact, in digital panoramic radiographs, the area situated inferiorly and laterally to the hyoid bone, corresponds to the bifurcation of the internal and external carotid arteries, which is exactly where carotid artery calcifications (CAC) are often present. In panoramic radiographs, the bifurcation of the internal and external carotids is situated around the intervertebral discs $\mathrm{C} 3$ and $\mathrm{C} 4$ or at a $45^{\circ}$ to the jaw angle $[2,3]$. For this reason, nodular and heterogeneous radiopacities found in these areas may be identified as atherosclerotic calcifications of the carotid arteries. Nonetheless, false-positive results are still possible, because other radiopaque structures are present in these areas, like the triticeous cartilage [4].

In the present study, irregular and heterogeneous radiopacities, occurring at the bifurcation of the carotid arteries, at the level of $\mathrm{C} 3-\mathrm{C} 4$, adjacent to the cervical spine and hyoid bone, were diagnosed as carotid artery calcifications (CAC).

The position of every CAC was also recorded: unilateral (right side or left side) or bilateral.

In all the positive findings, the patients' age and sex were also assessed.

(b) Elongated stylohyoid process
When an elongated stylohyoid process (ESP) was observed, its length was measured, according to the study of Jung et al. [5]. According to his study, the measurements are performed from the frontal view of the styloid process, where it leaves the tympanic plate of the temporal bone. The panoramic radiograph in this area presents a thin transparent line between the styloid process and the tympanic bone, which corresponds to the bone cleft present. The starting point of measurement (A) is situated where this radiolucency fades and the styloid process starts. On the other hand, the ending point of the measurement (B), is the radiopaque tip of the styloid process. The length of the line going from A to $B$ is considered the length of the elongated styloid process.

The lengths of these mineralized areas were measured using the electronic ruler and recorded.

Values higher than $25 \mathrm{~mm}$ were adopted as the manifestation of an elongated stylohyoid process. The position of the ESP was recorded as well: bilateral or unilateral (right side or left side). In the end, ESPs where broken into two categories: segmented and non-segmented. If no distinction between the stylohyoid ligament complex and the ossified stylohyoid ligament was possible, the entire osseous length of the stylohyoid apparatus was measured as a single unit and was considered non-segmented. Otherwise, if the ESP appeared fragmented, the segments were individually measured and then added together, obtaining the total osseous length.

(c) Maxillary sinus pathologies

The maxillary sinus area of the panoramic radiographs was then critically analyzed to identify pathological radiolucencies or radiopaque formations. For all of them, we recorded the position (right side, left side or bilateral), patients' age and sex.

(d) Other incidental findings

The panoramic radiographs included in the study were analyzed to identify all the other entities that did not appear as 'normal' and could not be included in the previous categories. All these were considered "other incidental findings". To correctly diagnose every single entity, differential diagnosis between them was made.

All procedures followed were in accordance with the ethical standards of the responsible committee on human experimentation (institutional and national) and with the Helsinki Declaration of 1975, as revised in 2008.

Informed consent was obtained from all patients for being included in the study, Additional informed consent was obtained from all patients for which identifying information is included in this article. 
Table 1 Characteristics of the analyzed population

\begin{tabular}{llllll}
\hline $\begin{array}{l}n \text {. Total } \\
\text { patients }\end{array}$ & $n$. males & $n$. females & $\%$ Males & $\%$ Females & $\begin{array}{l}\text { Average } \\
\text { age }\end{array}$ \\
\hline 2017 & 948 & 1069 & 47.00 & 53.00 & 47 \\
\hline
\end{tabular}

\section{Statistical analysis}

After data collection, we found the prevalence of patients presenting incidental findings. We then calculated the percentage of female and male patients, and the mean age in every category. We also studied the side distribution of every single finding.

The population was divided into 7 groups, according to the length of the ESP:

\begin{tabular}{ll}
\hline Group & ESP length $(\mathrm{mm})$ \\
\hline I & $\leq 25$ \\
II & {$[25 ; 30]$} \\
III & {$[30 ; 40]$} \\
IV & {$[40 ; 50]$} \\
V & {$[50 ; 60]$} \\
VI & {$[60 ; 80]$} \\
VII & $\geq 80$ \\
\hline
\end{tabular}

Each group was then further divided into 5 subgroups based on the age: $<20$ years; $20-39$ years; $40-59$ years; $60-79$ years; $\geq 80$ years. In every single group, the prevalence of female and male population was calculated.

\section{Results}

A total of 2307 panoramic radiographs were collected. According to the inclusion and the exclusion criteria, a final sample of 2017 radiographs was reached, with 1069 females (53\%), 948 males (47\%) and a ratio of 1:1.13 males to females. These individuals were aged between 6 and 91 years $($ mean $=47 ; \mathrm{SD}=16.25)$ Table 1.

(a) Carotid artery calcifications

Of these 2017 subjects, 167 (8.28\%) were found to have radiopaque lesions that were identified as CAC.

The 167 individuals with CAC consisted of 105 females $(62.87 \%)$ and 62 males $(37.13 \%)$, with a prevalence of $9.82 \%$ in women and $6.54 \%$ in men.

By analyzing the significance of the chi-square test, we observed that gender had a statistically significant association with the presence of CAC. In fact, females presented a greater risk of radiographic presentation of CAC than men Table 2.

The mean age of these patients was 65.33 years (men: 64.35, women: 65.90). There was no significant difference in mean age between men and women Table 3.

Of these 167 cases of CAC, 81 (48.5\%) were bilateral (52 females, 29 males), 32 (19.16\%) were located on the left side (21 females, 11 males) and $54(32.34 \%)$ were located on the right side ( 32 females, 22 males).

The prevalence of bilateral CAC was significantly higher than the prevalence of CAC on the right and the left side, for both male and female patients Fig. 1 .

\section{Elongated stylohyoid process}

Of the 2017 radiographs included in the study, 255 (12.64\%) displayed visible elongated stylohyoid processes (ESP). A total of 470 ESP were assessed in these 255 panoramic radiographs.

The prevalence of ESP in men was $13.82 \%$, while in women, it amounted to $11.60 \%$ Table 4.

Of these 255 subjects presenting ESP, 216 (84.71\%) were bilateral, while $20(7.84 \%)$ were on the right and $19(7.45 \%)$ were on the left side.

Of the 216 bilateral ESP, 113 (44.31\%) were males and $103(40.39 \%)$ were females.

Table 2 CAC prevalence

\begin{tabular}{llllllll}
\hline Carotid Artery Calcification & $n$. Total & Prevalence $(\%)$ & $n$. Males & Male prev (\%) & $n$. Females & Female prev $(\%)$ & Average age \\
\hline & 167 & 8.28 & 62 & 6.54 & 105 & 9.82 & 65.33 \\
\hline
\end{tabular}

Table 3 CAC distribution

\begin{tabular}{llllllll}
\hline \multicolumn{7}{l}{ Carotid Artery Calcification } \\
\cline { 2 - 7 } & $n$. Total & Bilateral & Right & Left & Bilateral (\%) & Right (\%) & Left (\%) \\
\hline Total & 167 & 81 & 54 & 32 & 48.50 & 32.34 & 19.16 \\
Males & 62 & 29 & 22 & 11 & 46.78 & 35.48 & 17.74 \\
Females & 105 & 52 & 32 & 21 & 49.52 & 30.48 & 20 \\
\hline
\end{tabular}


Fig. 1 Bilateral carotid artery calcifications at the level of the third cervical vertebra. Superior horn of thyroid cartilage and triticeous cartilage observable at the level of the fourth cervical vertebra

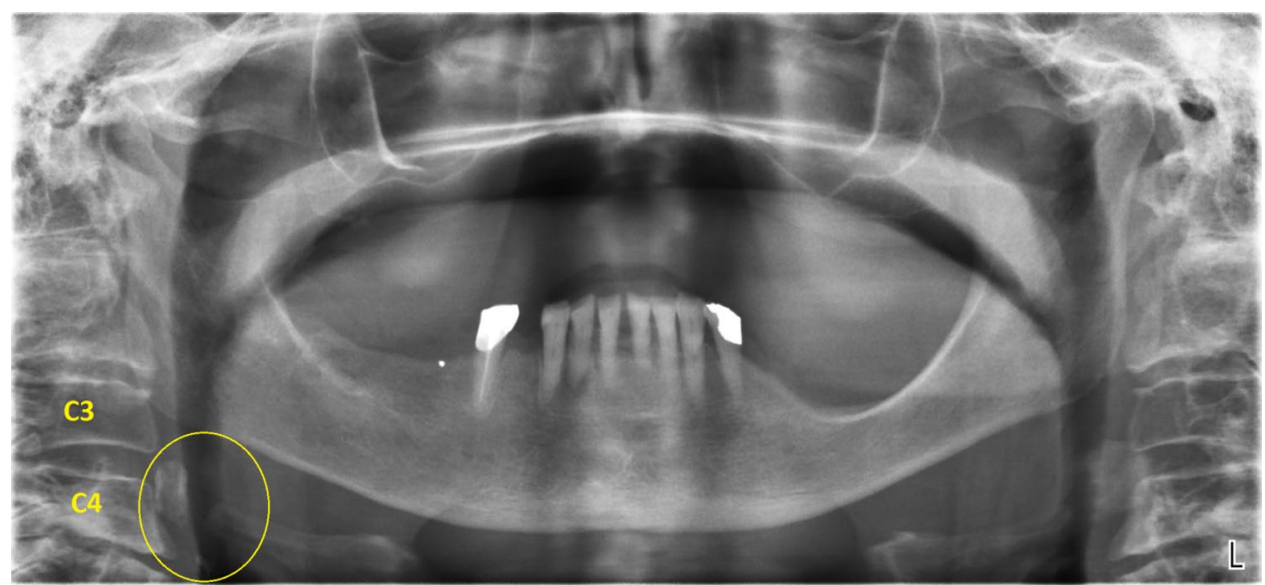

Table 4 ESP prevalence

\begin{tabular}{lllllll}
\hline & $n$. Total & Prevalence $(\%)$ & $n$. Males & Male prev (\%) & $n$. Females & Female prev (\%) \\
\hline Elongation of stylohyoid process & 255 & 12.64 & 131 & 13.82 & 124 & 11.60 \\
\hline
\end{tabular}

Table 5 ESP location and type, relating to sex

\begin{tabular}{llllllll}
\hline \multicolumn{7}{c}{ Elongation of Stylohyoid Process } \\
\cline { 2 - 7 } & $n$. Total & Bilateral & Right & Left & Bilateral (\%) & Right (\%) & Left (\%) \\
\hline Total & 255 & 216 & 20 & 19 & 84.71 & 7.84 & 7.45 \\
Males & 131 & 113 & 7 & 11 & 44.31 & 2.75 & 4.31 \\
Females & 124 & 103 & 13 & 8 & 40.39 & 5.10 & 3.14 \\
\hline
\end{tabular}

Table 6 ESP location and type, relating to sex

\begin{tabular}{llllcl}
\hline & \multicolumn{2}{l}{ Elongation of Stylohyoid Process } & & \\
\cline { 2 - 4 } & $n$. Total & Segmented & Non-segmented & Segmented (\%) & $\begin{array}{l}\text { Non- } \\
\text { segmented } \\
(\%)\end{array}$ \\
\hline Total & 255 & 34 & 221 & 13.33 & 86.67 \\
Males & 131 & 24 & 107 & 9.41 & 41.96 \\
Females & 124 & 10 & 114 & 3.92 & 44.71 \\
\hline
\end{tabular}

$13(5.10 \%)$ out of the $20 \mathrm{ESP}$ on the right side, were females, while $7(2.75 \%)$ were males. In the end, $11(4.31 \%)$ out of the 19 ESP on the left side were males, and $8(3.14 \%)$ were females.

Out of the 255 cases of ESP, $221(86.67 \%)$ were nonsegmented (114 females, 107 males) and only 34 (13.33\%) were segmented ( 24 males, 10 females).

Thus, the prevalence of the non-segmented ESP was significantly higher than the segmented one and the prevalence of bilateral ESP was significantly higher than the ones present on the right and the left side Tables 5 and 6 .

Concerning the length of the ESP, values higher than $25 \mathrm{~mm}$ were adopted as the manifestation of the elongated stylohyoid process. In particular, it should be noted that
255 PRs showed ESPs, but the total number of ESPs was 470 , given that some of them were unilateral, while others were bilateral.

Out of the identified 470 ESPs as many as $71.28 \%$ were found to have a pathological length, i.e. greater than $25 \mathrm{~mm}$.

$28.72 \%$, on the other hand had a length $\leq 25 \mathrm{~mm}$.

In the panoramic radiographs with pathological ESP length $(>25 \mathrm{~mm})$, the highest prevalence was found in the $30-40 \mathrm{~mm}$-long group (29.57\%), followed by the $25-30 \mathrm{~mm}$ long group (20.64\%).

There was a greater tendency for the abnormality (ESP $>25 \mathrm{~mm})$ in over 40 years old and male patients Table 7; Fig. 2. 


\section{Maxillary sinus pathologies}

Out of the 2017 PRs reviewed, 36 (1.78\%) displayed maxillary sinus pathologies: 22 men $(61.11 \%)$ and 14 women $(38.89 \%)$. Thus, we obtained a prevalence of $2.32 \%$ in men and $1.31 \%$ in women Table 8 .

The mean age of the patients presenting maxillary sinus pathology was 49.64 years, ranging from 11 to 81 years Table 9.

The major part of these findings $(91.67 \%)$ was unilateral, while just $3(8.33 \%$ ) were bilateral (see Tables 10 , $11)$.

\section{Other incidental findings}

A total number of 529 incidental findings were seen: 255 (48.2\%) were ESP, 167 were CAC (31.57\%), 36 were maxillary sinus pathologies (6.8\%) and 71 "other incidental findings" (13.42\%).

These included: tonsilloliths, sialoliths, residual cysts, radiolucent cyst-like lesions, radiopaque lesions, mixed radiopaque-radiolucent lesions, hyperostosis, foreign bodies of endodontic origin.

Hence, the prevalence of these other kinds of incidental findings was $3.52 \%$.

Table 7 ESP length relating to age and sex

\begin{tabular}{|c|c|c|c|c|c|c|c|c|c|c|c|c|c|c|c|c|c|c|c|c|c|c|c|c|c|c|c|}
\hline \multirow[b]{3}{*}{ Group } & \multirow[b]{3}{*}{ Length (mm) } & \multicolumn{4}{|c|}{$<20$ years } & \multicolumn{4}{|c|}{ 20-39 years } & \multicolumn{4}{|c|}{ 40-59 years } & \multicolumn{4}{|c|}{$60-79$ years } & \multicolumn{4}{|c|}{$\geq 80$ years } & \multicolumn{4}{|c|}{ Total } & \multirow[b]{3}{*}{ Total } & \multirow[b]{3}{*}{$\%$} \\
\hline & & \multicolumn{2}{|l|}{$F$} & \multicolumn{2}{|l|}{$M$} & \multicolumn{2}{|l|}{$F$} & \multicolumn{2}{|l|}{$M$} & \multicolumn{2}{|l|}{$F$} & \multicolumn{2}{|l|}{$M$} & \multicolumn{2}{|l|}{$F$} & \multicolumn{2}{|l|}{$M$} & \multicolumn{2}{|l|}{$F$} & \multicolumn{2}{|l|}{$M$} & \multicolumn{2}{|l|}{$F$} & \multicolumn{2}{|l|}{$M$} & & \\
\hline & & $R$ & $L$ & $R$ & $L$ & $R$ & $L$ & $R$ & $L$ & $R$ & $L$ & $R$ & $L$ & $R$ & $L$ & $R$ & $L$ & $R$ & $L$ & $R$ & $L$ & $R$ & $L$ & $R$ & $L$ & & \\
\hline$I$ & $\leq 25$ & 1 & 1 & 0 & 0 & 12 & 6 & 5 & 3 & 17 & 18 & 10 & 10 & 12 & 11 & 15 & 11 & 1 & 0 & 1 & 1 & 43 & 36 & 31 & 25 & 135 & 28.72 \\
\hline II & {$[25 ; 30]$} & 0 & 0 & 0 & 0 & 7 & 9 & 2 & 2 & 6 & 6 & 10 & 10 & 15 & 9 & 6 & 9 & 1 & 3 & 1 & 1 & 29 & 27 & 19 & 22 & 97 & 20.64 \\
\hline$I I I$ & {$[30 ; 40]$} & 0 & 0 & 0 & 0 & 3 & 4 & 7 & 10 & 11 & 13 & 13 & 17 & 11 & 14 & 15 & 13 & 2 & 1 & 2 & 3 & 27 & 32 & 37 & 43 & 139 & 29.57 \\
\hline$I V$ & {$[40 ; 50]$} & 0 & 0 & 0 & 0 & 2 & 5 & 3 & 3 & 6 & 2 & 13 & 9 & 5 & 4 & 7 & 10 & 1 & 2 & 2 & 2 & 14 & 13 & 25 & 24 & 76 & 16.17 \\
\hline$V$ & {$[50 ; 60]$} & 0 & 0 & 0 & 0 & 1 & 1 & 1 & 0 & 0 & 0 & 3 & 5 & 0 & 1 & 2 & 3 & 2 & 1 & 0 & 1 & 3 & 3 & 6 & 9 & 21 & 4.47 \\
\hline$V I$ & {$[60 ; 80]$} & 0 & 0 & 0 & 0 & 0 & 0 & 0 & 0 & 0 & 0 & 0 & 0 & 0 & 0 & 0 & 0 & 0 & 0 & 1 & 0 & 0 & 0 & 1 & 0 & 1 & 0.21 \\
\hline \multirow[t]{2}{*}{$V I I$} & $\geq 80$ & 0 & 0 & 0 & 0 & 0 & 0 & 0 & 0 & 0 & 0 & 0 & 0 & 0 & 0 & 0 & 0 & 0 & 0 & 1 & 0 & 0 & 0 & 1 & 0 & 1 & 0.21 \\
\hline & Total & 1 & 1 & 0 & 0 & 25 & 25 & 18 & 18 & 40 & 39 & 49 & 51 & 43 & 39 & 45 & 46 & 7 & 7 & 8 & 8 & 116 & 111 & 120 & 123 & 470 & 100 \\
\hline
\end{tabular}

Fig. 2 ESP measurement

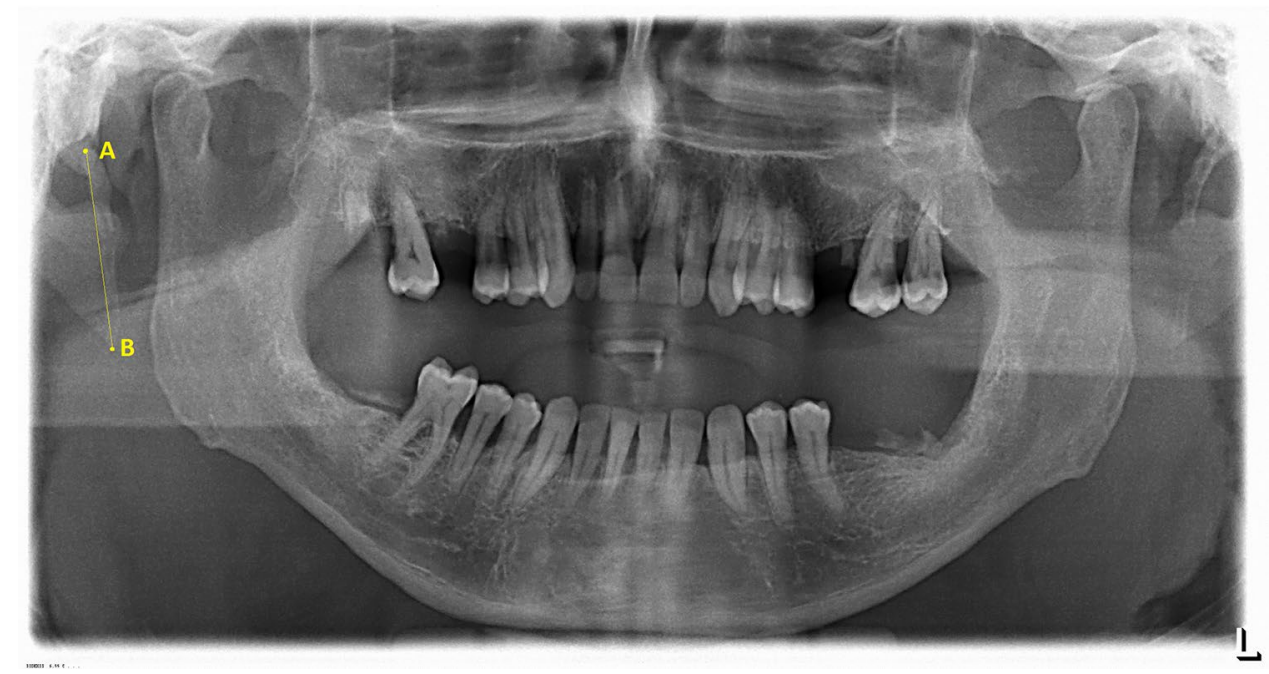

Table 8 Prevalence of maxillary sinus pathologies

\begin{tabular}{lllllll}
\hline & $n$. Total & Prevalence $(\%)$ & $n$. Males & Male pev (\%) & $n$. Females & Female prev (\%) \\
\hline Maxillary sinus pathology & 36 & 1.78 & 22 & 2.32 & 1.4 & 1.31 \\
\hline
\end{tabular}


Table 9 Maxillary sinus pathologies relating to sex and location

\begin{tabular}{|c|c|c|c|c|c|c|c|c|c|c|}
\hline & \multicolumn{10}{|c|}{ Maxillary sinus pathologies } \\
\hline & \multirow[t]{2}{*}{$n$. Total } & \multirow[b]{2}{*}{ Total } & \multicolumn{3}{|l|}{ Bilateral } & \multicolumn{3}{|l|}{ Right } & \multicolumn{2}{|l|}{ Left } \\
\hline & & & Females & Males & Total & Females & Males & Total & Females & Males \\
\hline Sinusitis & 22 & 3 & 1 & 2 & 11 & 5 & 6 & 8 & 3 & 5 \\
\hline Antral pseudocist & 14 & 0 & 0 & 0 & 13 & 5 & 8 & 1 & 0 & 1 \\
\hline Total & 36 & 3 & 1 & 2 & 24 & 10 & 14 & 9 & 3 & 6 \\
\hline
\end{tabular}

Table 10 Prevalence of other incidental findings

\begin{tabular}{llllllll}
\hline & $n$. Total & Prevalence $(\%)$ & $n$. Males & Male prev $(\%)$ & $n$. Females & Female prev $(\%)$ & Average age \\
\hline Other incidental findings & 71 & 3.52 & 45 & 4.75 & 26 & 2.43 & 54.17 \\
\hline
\end{tabular}

Table 11 Total prevalence and single prevalences of incidental findings

\begin{tabular}{lll}
\hline Incidental findings & Number & Prevalence (\%) \\
\hline Total & 529 & 26.23 \\
Carotid artery calcification & 167 & 8.28 \\
Elongation of stylohyoid process & 255 & 12.64 \\
Maxillary sinus pathology & 36 & 1.70 \\
Other incidental findings & 71 & 3.52 \\
\hline
\end{tabular}

Of these 71 patients, $45(63.38 \%)$ were men and 26 (36.62\%) were women, with a mean age of 54.17 years, ranging from 19 to 85 years.

The "other incidental findings" with the highest prevalence of occurrence were sialoliths and tonsilloliths, amounting, respectively, to 18 cases (25.35\%) and 15 cases (21.13\%). Hence, the prevalences of sialoliths and tonsilloliths were, respectively, $0.89 \%$ and $0.74 \%$.

We then found: 4 residual cysts ( 2 on the mandibular left side, 1 on the mandibular right side, 1 on the maxillary right side); 3 radiolucent cyst-like lesions ( 1 on the mandibular right side, 1 in the mandibular symphysis, 1 on the maxillary right side); 23 radiopaque lesions (1 on the maxillary left side and the other 22 occurring in the mandible: 9 on the left side, 7 on the right side, 6 in parasymphysis); 3 mixed radiopaque-radiolucent lesions ( 2 on the mandibular left side and 1 on the maxillary right side); 4 hyperostosis ( 3 occurring on the mandibular right side and 1 occurring in the mandibular left side); 2 small radiopaque lesions in the maxillary sinus, which appeared similar to foreign bodies of endodontic origin.

In conclusion, a total number of 529 incidental findings were seen: $48.2 \%$ of them were ESP, $31.57 \%$ were CAC,
Table 12 Percentage of the incidental findings

\begin{tabular}{lll}
\hline Incidental findings & Number & Percentage (\%) \\
\hline Carotid artery calcification & 167 & 31.57 \\
Elongation of stylohyoid process & 255 & 48.20 \\
Maxillary sinus pathology & 36 & 6.80 \\
Other incidental findings & 71 & 13.42 \\
\hline
\end{tabular}

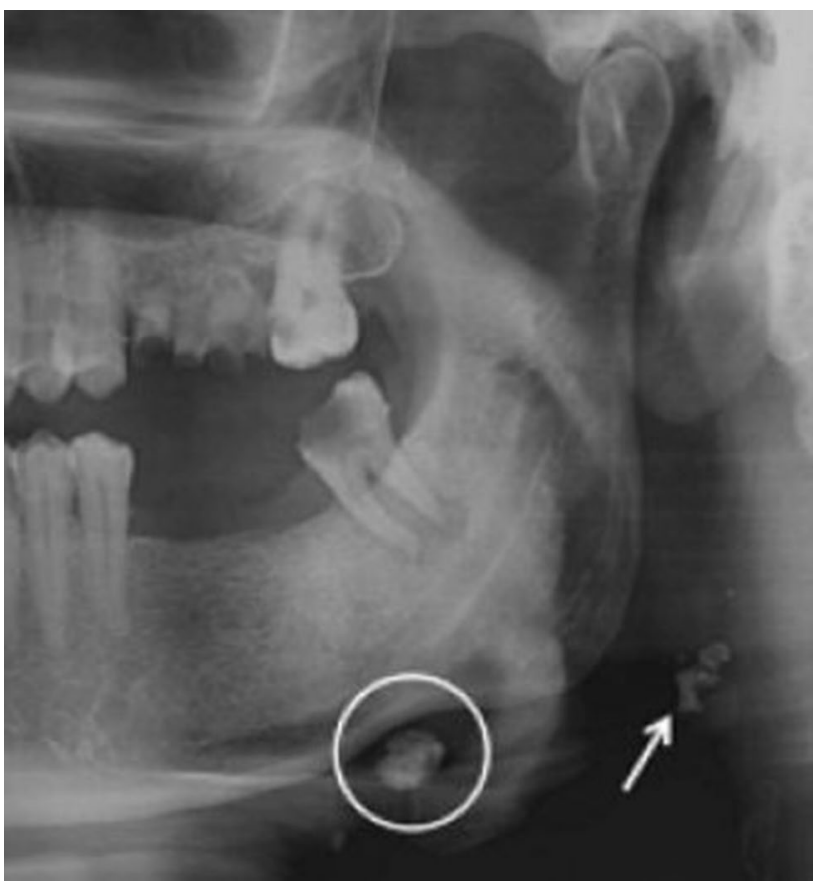

Fig. 3 A well-defined, solitary nodular radiopaque structure was seen inferior to the left mandibular border, near the angle. Apart from this, multiple punctuate radiopacities were seen at the level of the lower margin of the cervical vertebra on the left side. Based on the radiographic location and appearance of the lesion on the radiograph, a provisional diagnosis of the left submandibular gland sialolothiasis and carotid artery calcification was made 
$6.8 \%$ were maxillary sinus pathologies and $13.42 \%$ were other incidental findings.

The total prevalence of the incidental findings was 26,23\% Table 12; Fig. 3.

\section{Discussion}

A peculiarity of this study was that panoramic radiographs were analyzed for all kinds of incidental findings not related to dentistry. Both the single prevalence of each of them and the overall prevalence were thus obtained.

Also, MacDonald et al. [6] analyzed consecutive PRs of symptom-free patients, but these ones attended a Canadian general dental practice and solely for dental examination or hygiene. Therefore, this sample was not representative of the general population.

\section{Carotid artery calcification}

Cerebral vascular accidents represent the third cause of death in industrialized countries [2]. They are one of the major public health problems, because of their high incidence and the rehabilitation cost of patients [3].

This study showed that panoramic radiography could help to diagnose carotid artery calcifications.

An accurate analysis of PRs for carotid calcifications could be a useful tool in assessing individuals at risk for

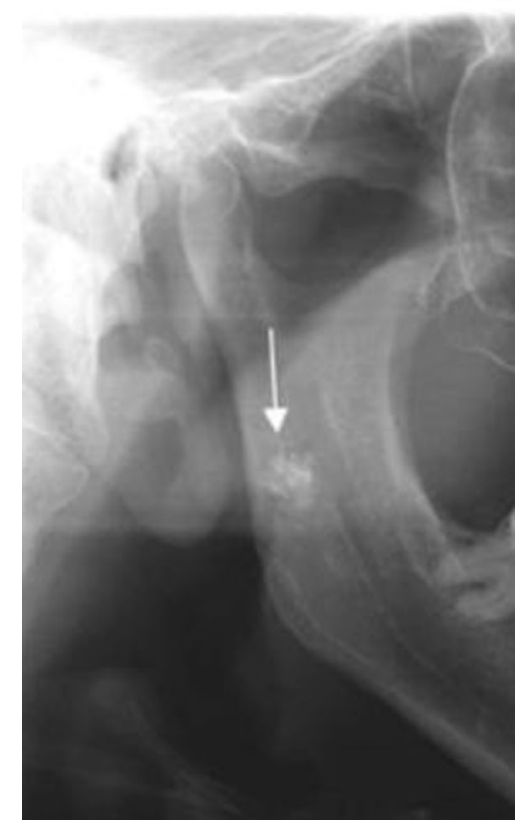

Fig. 4 Tonsilloliths are superimposed in the right mandibular ramus

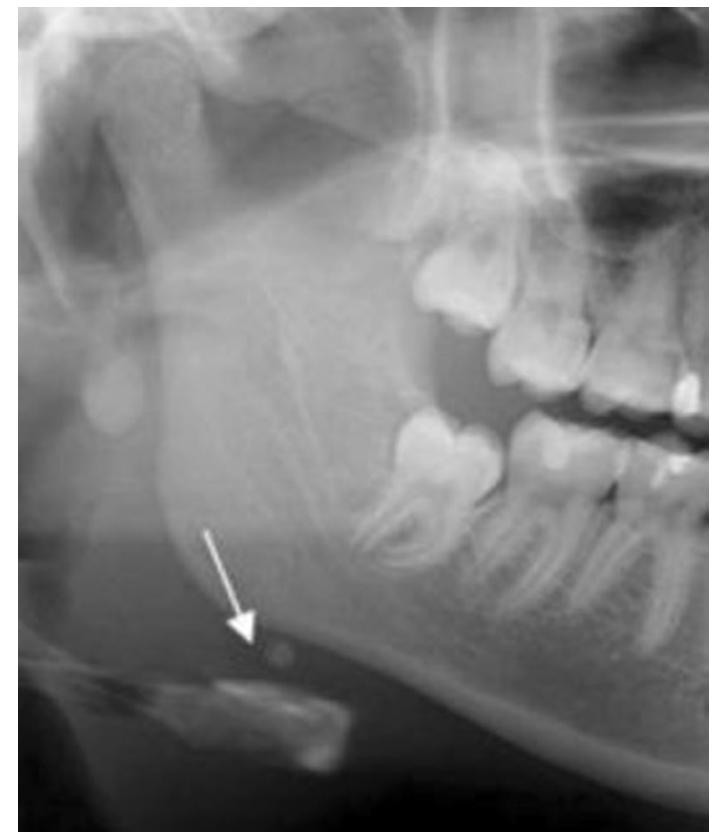

Fig. 5 A sialolith under right mandibular border

stroke, although it requires training to detect calcifications on panoramic radiographs Figs. 4, 5, 6, 7, 8, 9 and 10.

In literature, we can find some variance in the prevalence of CAC visible on the PRs.

This study found a prevalence of $8.28 \%$ of the presence of carotid artery calcifications on the panoramic radiographs, while Monsour [7] found calcifications in 4\% of a total of 2628 non-digital panoramic radiographs. However, our study, unlike the study by Monsour [7], uses digital panoramic radiographs, which enables low-density calcifications to be identified, also because the image contrast can be modified.

Dorado et al. [8] found a prevalence of $15.5 \%$, while Bryam et al. [9] obtained a result of $2.1 \%$.

Monteiro et al. [10] found a prevalence of $9.5 \%$, which is similar to the one found in the present study.

We detected CACs through panoramic radiography in $8.28 \%$ of the subjects (with an average age of 65.33 years), and most of them were women $(62.87 \%)$.

The result of women having a significantly higher prevalence of CAC (9.82\% vs a prevalence of $6.54 \%$ in men), may suggest a relationship between the decline of estrogen levels in the blood of postmenopausal women and CAC, as proposed by Friedlander and Altoman [11]. In fact, they reported that estrogen decreases low-density lipoprotein (LDL) catabolism in blood and that increased LDL cholesterol levels in blood were associated with the risk of cardiovascular pathologies. Although some studies have suggested an increased prevalence in women, this statistical significance has been contested (Levy and Mandel, [12]). 
Fig. 6 a, b Bilateral carotid artery calcifications at the level of the third cervical vertebra. Superior horn of thyroid cartilage and triticeous cartilage observable at the level of the fourth cervical vertebra
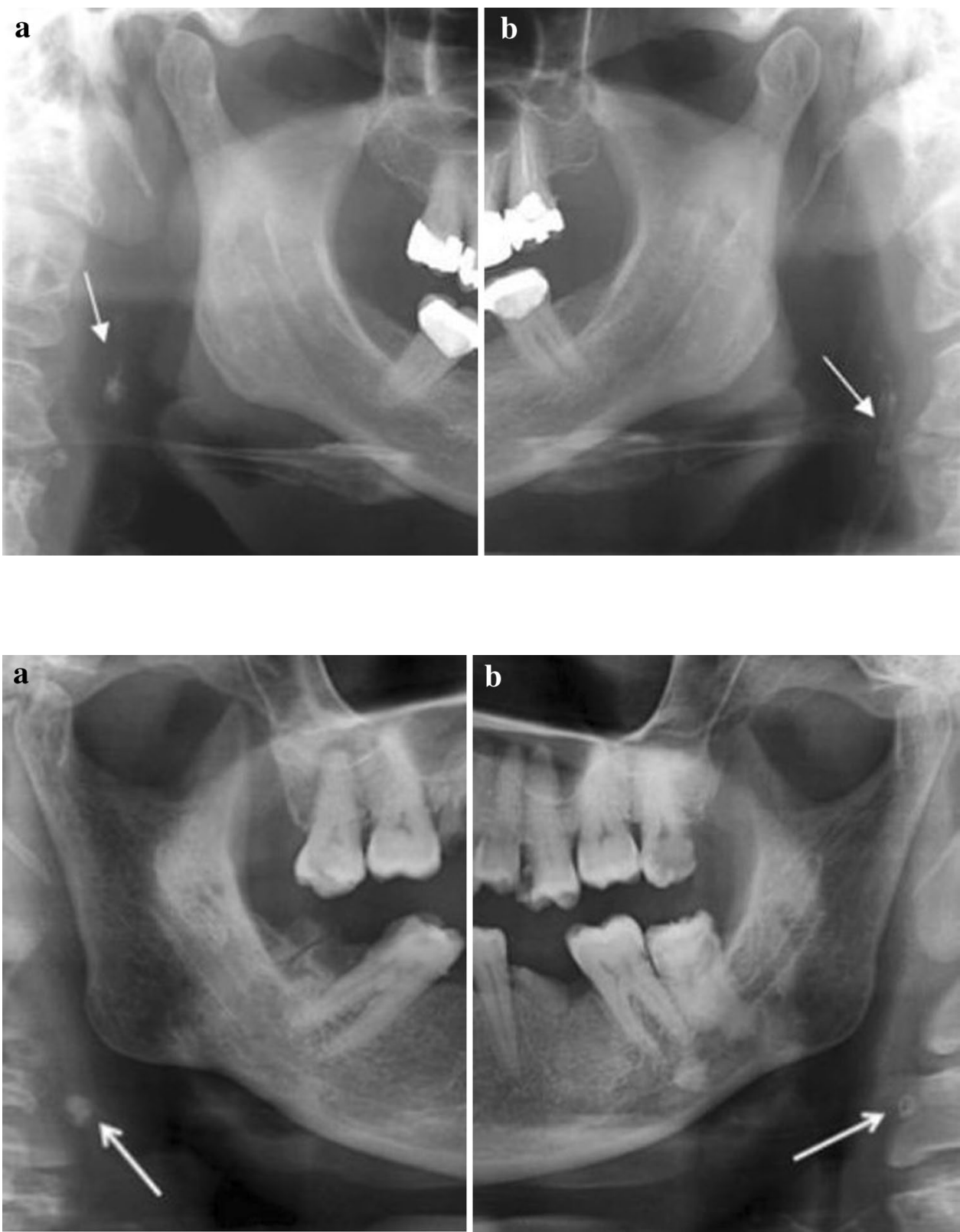

Fig. 7 a, b Well defined, bilateral nodular radiopacities were seen at the level of the lower margin of the third and the fourth cervical vertebra (C3 \& $\mathrm{C} 4)$. Based on the radiographic location and appearance of the lesion on the radiograph, a provisional diagnosis of the coronary artery calcification was made

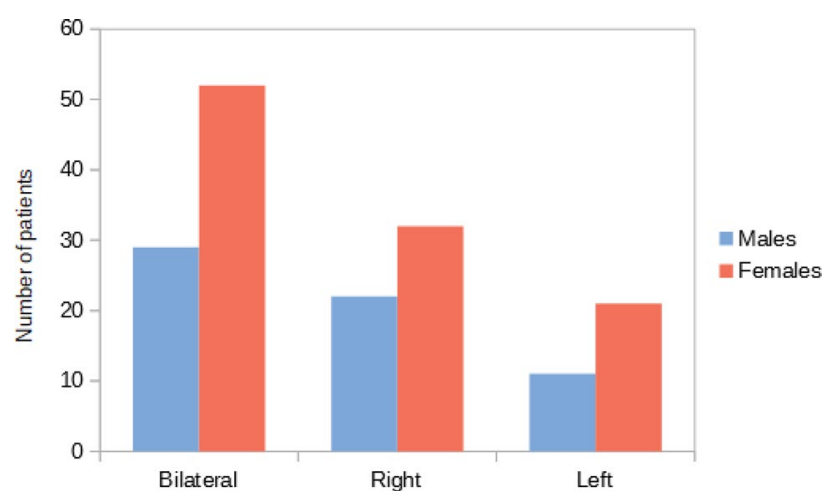

Fig. 8 CAC location relating to sex

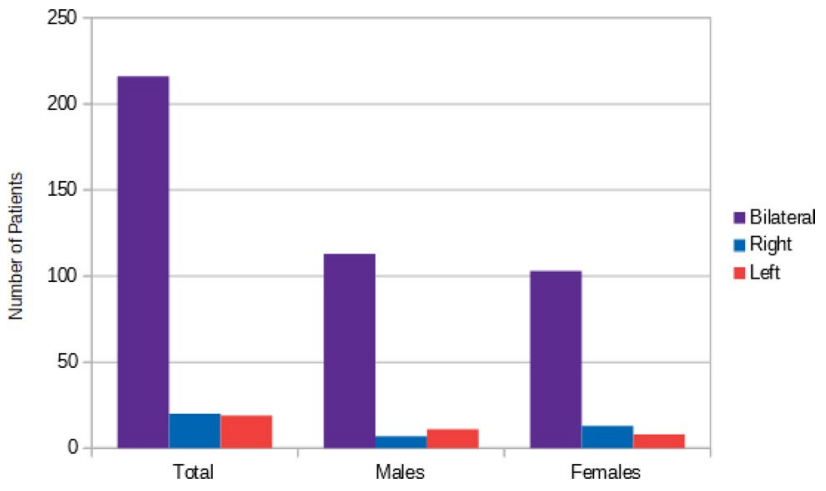

Fig. 9 ESP location 


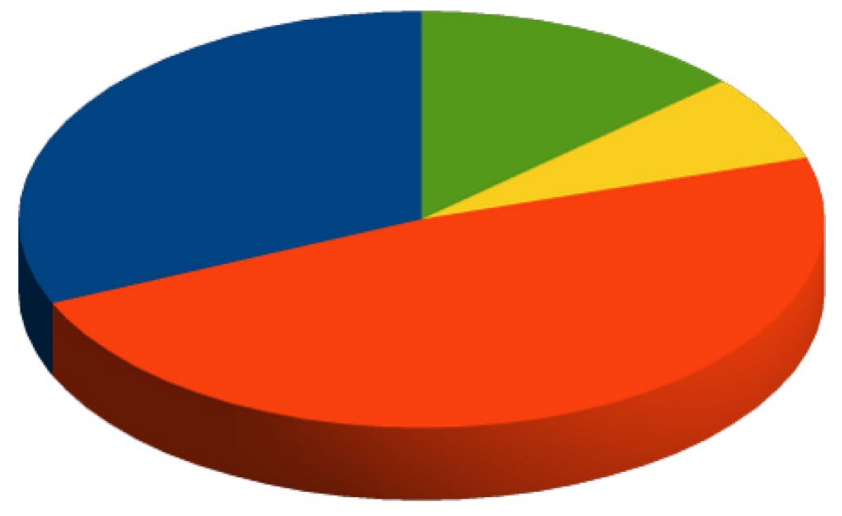

a Carotid Artery Calcification E Elongation of Stylohyoid Process naxillary Sinus Pathology = Other Incidental Findings

Fig. 10 Percentage of the incidental findings

The recognition of CAC on PR has some limitations, such as quantifying the degree of stenosis. Consequently, Doppler ultrasound is still the main diagnostic test of this pathology, being accurate and non-invasive $[13,14]$.

\section{Elongated stylohyoid process}

The measurement of the stylohyoid process on a panoramic radiograph was challenged. In fact, a 12 pattern classification, which displayed significant difference between prevalences of different patterns varied between East Asian and Western communities [15].

In our study, the prevalence of ESP was $12.64 \%$. This value is lower than that found by Rizzatti-Barbosa et al. [16].

Several studies have concluded that there is a large variation in the length of the styloid ligament complex, and this varies from individual to individual. In general, styloid processes $>25 \mathrm{~mm}$ are considered to be of abnormal length.

The results from the present study indicated that the length of $28.72 \%$ of the calcified stylohyoid processes that were found, was shorter than $25 \mathrm{~mm}$, while the remaining $71.28 \%$ was longer than $25 \mathrm{~mm}$.

In the panoramic radiographs with pathological ESP length $(>25 \mathrm{~mm})$, the highest prevalence was found in over 40 years old and male patients.

Concerning the spatial distribution of ESPs, our study, just like that of Rizzatti-Barbosa et al. [16], has shown that it could tend to their bilateral position.

Ultimately, the prevalence of the non-segmented ESPs was significantly higher than the segmented ones and the prevalence of bilateral ESPs was significantly higher than the ones present on the right and the left side.

In conclusion, as proposed by Steinmann [17], there are 3 theories to explain ossification of the stylohyoid process: the theory of reactive hyperplasia, the theory of reactive metaplasia, the theory of anatomic variation.

The latter involves the stylohyoid ligament or the styloid process as ossified structures that develop in the early formative years after birth. This theory may fit in radiographic findings of ossification in children and young adolescents [18]. However, our study showed that just 2 out of the 255 panoramic radiographs presenting ESP, were found in patients younger than 20 years old. Hence, this finding may demonstrate that the theory of anatomic variation proposed by Steinman [17] is not valid in our study.

\section{Maxillary sinus pathologies}

Although panoramic radiography is not considered the gold standard for the diagnosis of maxillary sinus pathologies and cannot be used to entirely exclude their presence, it can help to detect some of them. Being frequently used, it may help to identify particularly the asymptomatic ones or the most insidious ones. This may be crucial in the case of malignancies, because early identification can be very important for the prognosis. As reported in literature, early detection can result in at least $80 \%$ treatment success rate as determined by the 5 -year survival $[19,20]$. It has also been found that at the time of diagnosis of antral malignancies, these can be seen on panoramic radiographs in $90 \%$ of the cases [21].

Halstead et al. [22], reported that sinus pathologies detected with PR had a prevalence of about $2 \%$. Our study found a similar result, since $1.78 \%$ of the radiographs reviewed displayed maxillary sinus pathologies, indicating that PR may be a useful additional tool to detect maxillary sinus diseases. The mean age of these patients was 49 , 64 years and they were mostly men (61.11\%).

Concerning the spatial distribution, $91.67 \%$ of these pathological findings were unilateral, indicating that maxillary sinus diseases are most frequently present on a single side. In particular, the right side showed a higher incidence.

Lastly, antral pseudocyst and maxillary sinusitis showed the highest prevalence, respectively, representing $36.11 \%$ and $30.56 \%$ of the maxillary sinus pathologies.

The literature reports that the prevalence of mucosal thickening from an inflammatory origin (such as antral pseudocyst and true sinus mucocele), averages around 38.89\%, but varies considerably from report to report, perhaps as a function of population, geography and season [22, 23].

Our study included panoramic radiographs performed between December 2013 and June 2016. Hence, all the seasons were included, allowing us to collect a more homogeneous data set.

Compatibly with the literature [22-24], our study found a similar prevalence of maxillary sinus inflammatory diseases, since $38.78 \%$ of the sinus pathologies found, were assessed 
as antral pseudocysts, while the remaining cases appeared as maxillary sinusitis.

\section{Other incidental findings}

The other incidental findings encountered included: sialoliths, tonsilloliths, residual cysts, radiolucent cyst-like lesions, radiopaque lesions, mixed radiopaque-radiolucent lesions, hyperostosis and foreign bodies of endodontic origin.

The prevalence of these occasional findings was $3.52 \%$ and the most frequently seen ones were sialoliths and tonsilloliths, with a prevalence of $0.89 \%$ and $0.74 \%$, respectively.

According to Mandel [25], tonsilloliths affect male and female patients equally, while Suarez-Cunqueiro et al. [26] described a greater occurrence in adult males (1.6:1).

Our study, congruently to Suarez-Cunqueiro et al. [26], found a higher prevalence in male patients (ratio $=1.25: 1$ ).

\section{Conclusion}

This study demonstrated the usefulness of panoramic radiography in showing a broad range of different pathological conditions not strictly related to dentistry, such as carotid artery calcifications, elongated stylohyoid processes, maxillary sinusitis, antral pseudocysts, sialoliths, tonsilloliths, etc. Due to the high prevalence of occasional findings, dental practitioners should be aware of the various conditions that may be encountered.

In particular, the widespread recognition that calcifications seen in the region of the carotid bifurcation can identify a population with a higher risk of stroke, supports the practice of routinely examining this area.

Since a panoramic radiograph is usually performed for dental reasons, an in-depth examination of the image is substantially cost-free and can help to identify unknown pathologies and, thus, prolong lives and bring significant savings in overall healthcare costs.

Therefore, dentists should be able to recognize the widest range of occasional findings that could be found in the PRs, to allow early medical interventions.

Funding Open access funding provided by Università degli Studi di Padova within the CRUI-CARE Agreement.

\section{Compliance with ethical standards}

Conflict of interest The authors declare that they have no conflict of interest.
Open Access This article is licensed under a Creative Commons Attribution 4.0 International License, which permits use, sharing, adaptation, distribution and reproduction in any medium or format, as long as you give appropriate credit to the original author(s) and the source, provide a link to the Creative Commons licence, and indicate if changes were made. The images or other third party material in this article are included in the article's Creative Commons licence, unless indicated otherwise in a credit line to the material. If material is not included in the article's Creative Commons licence and your intended use is not permitted by statutory regulation or exceeds the permitted use, you will need to obtain permission directly from the copyright holder. To view a copy of this licence, visit http://creativecommons.org/licenses/by/4.0/.

\section{References}

1. Friedlander AH, Lande A. Panoramic radiographic identification of carotid arterial plaques. Oral Surg Oral Med Oral Pathol. 1981;52:102-4.

2. Chicano R, Sanchez R, Castano F, Merino M, Lopez F. Panoramic radiograph as a method for detecting calcified atheroma plaques Review of lierature. Med Oral Patol Oral Cir Bucal. 2006;11:E261-6.

3. Macdonald D, Chan A, Harris A, Vertinsky T, Farman A, Scarfe W. Diagnosis and management of calcified carotid artery atheroma: dental perspectives. Oral Surg Oral Med Oral Pathol Oral Radiol. 2012;114:533-47.

4. Wakabayashi I. Associations between alcohol drinking and multiple risk factors for atherosclerosis in smokers and nonsmokers. Angiology. 2010;61(5):495-503.

5. Jung T, et al. Elongated styloid process: when is it really elongated? Dentomaxillofac Radiol. 2004;33:119-24.

6. MacDonald D, Yu W. Incidental findings in a consecutive series of digital panoramic radiographs. Imaging Sci Dent. 2020;50(1):53-64. https://doi.org/10.5624/isd.2020.50.1.53.

7. Monsour P, Young W. Variability of the styloid process and stylohyoid ligament in panoramic radiographs. Oral Surg Oral Med Oral Pathol. 1986;61(5):522-6.

8. Dorado C, Bonet C, Berrocal I, Caliz F, Gonzalez J. Relation between diagnosis of atheromatous plaque from orthopantomographs and cardiovascular risk factors. A study of cases and control subjects. Med Oral Patol Oral Cir Bucal. 2016;21(1):e66-71.

9. Bayram B, Uckan S, Acikgoz A, Muderrisoglu H, Aydinalp A. Digital panoramic radiography: a reliable method to diagnose carotid artery atheromas? Dentomaxillofac Radiol. 2006;35(4):266-70.

10. Monteiro IA, Ibrahim C, Albuquerque R, Donaldson N, Salazar F, Monteiro L. Assessment of carotid calcifications on digital panoramic radiographs: Retrospective analysis and review of the literature. J Stomatol Oral Maxillofac Surg. 2018;119(2):102-6.

11. Friedlander AH, Altman L. Carotid artery atheromas in postmenopausal women. Their prevalence on panoramic radiographs and their relationship to atherogenic risk factors. J Am Dent Assoc. 2001;132(8):1130-6.

12. Levy C, Mandel L. Calcified carotid artery imaged by computed tomography. J Oral Maxillofac Surg. 2010;68(1):218-20.

13. Shah S, Casas JP, Drenos F, Whittaker J, Deanfield J, Swerdlow DI, Holmes MV, Kivimaki M, Langenberg C, Wareham N, Gertow K, Sennblad B, Strawbridge RJ, Baldassare D, Veglia F, Tremoli E, Gigante B, de Faire U, Kumari M, Talmud PJ, Hamsten A, Humphries SE, Hingorani AD. Causal relevance of blood lipid fractions in the development of carotid atherosclerosis Mendelian randomization analysis. Circ Cardiovasc Genet. 2013;6(1):63-72. 
14. Giudice R, Izzo R, Manzi MV, Pagnano G, Santoro M, Rao MA, Di Renzo G, De Luca N, Trimarco V. Lifestyle-related risk factors, smoking status and cardiovascular disease. High Blood Press Cardiovasc Prev. 2012;19(2):85-92.

15. MacDonald-Jankowski DS. Calcification of the stylohyoid complex in Londoners and Hong Kong Chinese. Dentomaxillofac Radiol. 2001;30(1):35-9. https://doi.org/10.1038/sj/dmfr/46005 74.

16. Rizzatti-Barbosa CM, Ribeiro MC, Silva-Concilio LR, Di Hipolito O, Ambrosano GM. Is an elongated stylohyoid process prevalent in the elderly? A radiographic study in a Brazilian population. Gerodontology. 2005;22(2):112-5.

17. Steinmann EP. Styloid syndrome in absence of an elongated process. Acta Otolaryngol. 1968;66(4):347-56.

18. Camarda AJ, Deschamps C, Forest D. Stylohyoid chain ossification: A discussion of etiology. Oral Surg Oral Med Oral Pathol. 1989;67(5):515-20.

19. Hayashi T, Nonaka S, Bandoh N, Kobayashi Y, Imada M, Harabuchi Y. Treatment outcome of maxillary sinus squamous cell carcinoma. Cancer. 2001;92(6):1495-503.

20. Tiwari R, Hardillo JA, Mehta D, Slotman B, Tobi H, Croonenburg E, van der Waal I, Snow GB. Squamous cell carcinoma of maxillary sinus. Head Neck. 2000;22(2):164-9.

21. Epstein JP, Waisglass M, Bhimji S, Le N, Stevenson-Moore P. A comparison of computed tomography and panoramic radiography in assessing malignancy of the maxillary antrum. Eur J Cancer Oral Oncol. 1996;32B(3):191-201.

22. Halstead CL. Mucosal cysts of the maxillary sinus: report of 75 cases. J Am Dent Assoc. 1973;87(7):1435-41.

23. MacDonald-Jankowski DS. Mucosal antral cysts in a Chinese population. Dentomaxillofac Radiol. 1993;22(4):208-10.

24. Bohay RN, Gordon SC. The maxillary mucous retention cyst: a common incidental panoramic finding. Oral Health. 1997;87(7):7-10.

25. Mandel L. Multiple bilateral tonsilloliths: case report. J Oral Maxillofac Surg. 2008;66(1):148-50.

26. Suarez-Cunqueiro MM, Duker J, Liebehenschel N, Schön R, Schmelzeisen R. Calcification of the branches of the external carotid artery detected by panoramic radiography: a case report. Oral Surg Oral Med Oral Pathol Oral Radiol Endod. 2002;94(5):636-40.

Publisher's Note Springer Nature remains neutral with regard to jurisdictional claims in published maps and institutional affiliations. 